\title{
4D Scanning Transmission Electron Microscopy of a Twisted WS 2 Multilayer Structure
}

\author{
Chenyu Zhang, Yuzhou Zhao, Song Jin and Paul Voyles
}

University of Wisconsin-Madison, Madison, Wisconsin, United States

Stacking layers of two-dimensional materials create an additional periodicity longer than the unit cell arising from the Moire interference pattern of the twisted lattices, which can give rise to a variety of unconventional behaviors and properties ${ }^{1}$. Here we apply 4D scanning transmission electron microscopy (4D STEM $)^{2}$ to study a twisted multilayer $\mathrm{WS}_{2}$ structure that acquires its twist from geometric constraints during synthesis, rather than artificially through layer stacking. Convergent beam electron diffraction (CBED) patterns acquired from 4D STEM were used to measure the lattice twist angle between layers and show that it is consistent with the geometrically-required morphological twist. High-resolution STEM images visualize Moiré patterns ${ }^{3}$ consistent with the twist angle from diffraction.

Dislocation-driven spiral structures of WS2 were synthesized by water vapor assisted chemical vapor transport method ${ }^{4}$. Conditions were introduced to constrain these spirals onto curved geometry. Such geometry requires a consistent twist of each spiral layer in the morphology, which should increase linearly with the number of layers. We hypothesize that the morphological twist is tracked by the same twist in the crystal lattice.

A Thermo-Fisher Titan operating at $200 \mathrm{kV}$ equipped with a DE-16 direct electron camera was used for the 4D STEM experiment. An electron beam with a $0.6 \mathrm{mrad}$ semi-convergence angle was used to scan across the multilayer structure to get well-separated diffraction spots from each layer. The DE-16 camera was operated at 20 frames per second to ensure a signal at a low beam current of $0.4 \mathrm{pA}$, adopted to avoid damage to the $\mathrm{WS}_{2}$. Position averaged CBED (PACBED) patterns were formed by averaging CBED patterns over the regions with 1, 2, 3, and 4 layers of $\mathrm{WS}_{2}$. The PACBED patterns are shown in Figure 1a-d. Lattice twist angles were measured on PACBED patterns using all six diffraction spots. The geometric twist angle was measured from the low magnification STEM image shown in figure 1e. Twist angles from two different measurements are plotted in figure 1f. Two separate measurements agree well with each other, and both show a linear relationship between twist angle and number of layers as expected from the growth mechanism.

High angle annular dark-field (HAADF) STEM images were collected on the same structure with a 24.5 mrad convergence angle and sub- $\AA$ spatial resolution. HAADF images from 1-, 2-, and 3-layer areas are shown in figure $2 \mathrm{a}-\mathrm{c}$, after high-pass filtering to emphasize the lattice contrast and remove contrast from surface contamination. The 1-layer area shows a clear single crystal WS2 structure, and the 2-, 3-layer areas show Moiré patterns. Model Moiré patterns shown in figure $2 \mathrm{~d}$-f are constructed by overlapping multiple layers of $\mathrm{WS}_{2}$ structure with a twist angle taken from diffraction measurements and a translation was added to match the experiment. The model and experimentally collected Moiré patterns match well with each other, again confirming the lattice twist angle measurement from 4D STEM. 

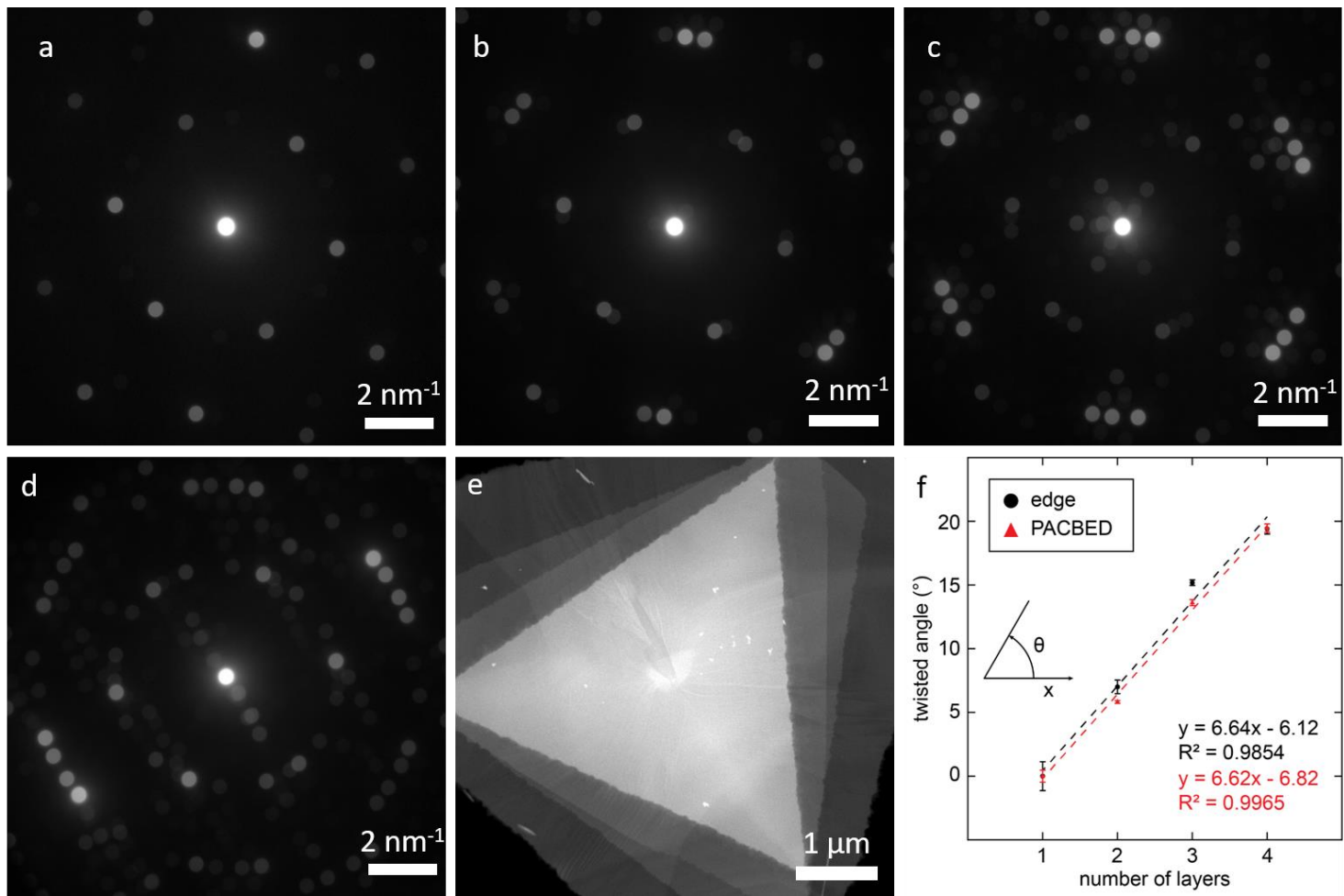

Figure 1. a-d, PACBED patterns generated from 1-, 2-, 3-, and 4-layer area on the twisted structure with a small semi-convergence angle. e, overall geometric shape of the whole sample containing four layers of WS2. f, plots of twist angle measured from PACBED patterns (red) and geometric shape (black) vs. the number of layers.
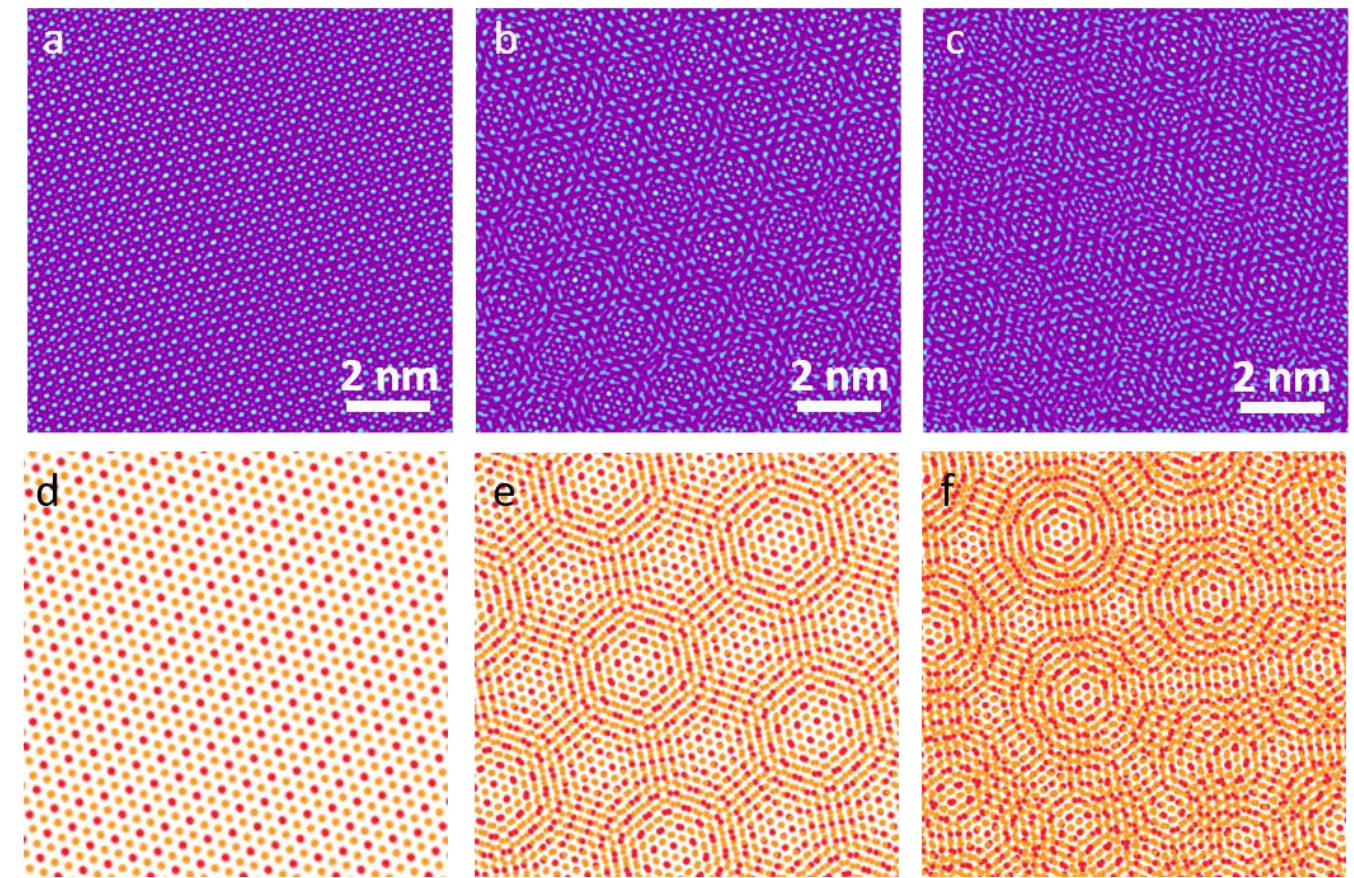

Figure 2. a-c, false color HAADF images acquired from 1-, 2-, and 3-layer area on the twisted structure with clear Moiré patterns shown in $b$ and c. d-f expected high-resolution images and Moiré patterns 
constructed by overlapping 1, 2, and 3 layers of WS2 structure with twist measured from PACBED patterns. Red dots represent $\mathrm{W}$ atoms, and yellow dots represent $\mathrm{S}$ atoms.

\section{References}

1. Cao, Y. et al. Unconventional superconductivity in magic-angle graphene superlattices. Nature 556, 43-50 (2018).

2. Ophus, C. Four-Dimensional Scanning Transmission Electron Microscopy (4D-STEM): From Scanning Nanodiffraction to Ptychography and Beyond. Microsc. Microanal. 25, 563-582 (2019).

3. Liu, Y. et al. Helical van der Waals crystals with discretized Eshelby twist. Nature 570, 358-362 (2019). 4. Zhao, Y. \& Jin, S. Controllable Water Vapor Assisted Chemical Vapor Transport Synthesis of WS 2 MoS 2 Heterostructure . ACS Mater. Lett. 2, $42-48$ (2020). 